Artigo

\title{
Diagnóstico laboratorial de hemoglobinopatias em populações diferenciadas
}

Giselda M. Orlando
Paulo C. Naoum

Fatima A. M. Siqueira ${ }^{3}$

Cláudia R. Bonini-Domingos ${ }^{4}$
As hemoglobinopatias são um grupo heterogêneo de distúrbios herdados recessivamente que incluem as talassemias e as doenças falciformes. As mutações que as originam são específicas de algumas regiões e em muitos casos determinadas por distribuições étnicas e geográficas, fundamentando os programas de controle destas alterações e o aconselhamento genético. 0 diagnóstico de alterações de hemoglobinas envolve cuidados com a metodologia aplicada eo grupo populacional que será avaliado. A informação sobre o tipo de hemoglobina alterada e o suporteclínico, psicológico e genético ao portador e seus familiares é de grande importância para o sucesso de programas preventivos nesta área. Com objetivo de avaliar as metodologias disponíveis para o diagnóstico laboratorial das hemoglobinopatias e suas aplicações em laboratórios clínicos, comparamos a incidência de hemoglobinas anormais em populações diferenciadas a saber: doadores de sangue, portadores de anemia a esclarecer, recém-nascidos, e estudantes. As metodologias aplicadas envolveram procedimentos eletroforéticos, análises citológicas e bioquímicas de triagem e para confirmação. No período de setembro de 1999 a janeiro de 2000 analisamos 524 indi víduos, com presença de formas variadas de alteração de hemoglobina para cada grupo, sendo que, dentre as amostras da população de doadores de sangue, foram diagn osticados doiscasos deindivíduos heterozigotos para anemia falciforme. Rev.bras.hematol.hemoter., 2000, 22(2): 111-121

Palavras-chave: preven ção de hemoglobinopatias, diagnóstico laboratorial, hemoglobinas anormais, talassemias

\section{Introdução}

As hemoglobinopatias, também conhecidas como distúrbios hereditários da hemoglobina humana, são doenças geneticamente determinadas e apresentam morbidade significativa em to do o mundo (1). Milhões de pessoas trazem em seu patrimônio genético, hemoglobinas anormais em várias combinações com conseqüências que variam das quase imperceptíveis às letais. Dessa forma, as anemias hereditárias compreendem

1 - Instituto de Biociências, UNESP - Botucatu, SP

2 - Professor Titular do Instituto de Biociências, Letras e Ciências Exatas, UNESP - São José do Rio Preto, SP

3 - Instituto de Biociências Letras e Ciências Exatas, UNESP - São José do Rio Preto, SP

4 - Bióloga do Instituto de Biociências, Letras e Ciências Exatas, UNESP - São José do Rio Preto, SP

Trabalho desenvolvido no Laboratório de Hemoglobinas, Departamento de Biologia, Instituto de Biociências, Letras e Ciências Exatas, UNESP - São José do Rio Preto, SP

Correspondência para: Dra. Cláudia Regina Bonini-Domingos Laboratório de Hemoglobinas, UNESP

Rua Cristóvão Colombo, 2265. CEP: 15054-000. São José do Rio Preto. SP

Fone: (17) 221-2392. Fax: (17) 221-2390. E-mail: bonini@bio.ibilce.unesp.br 
um grupo de condições de considerável complexidade (2).

O Brasil se caracteriza por significativa mistura racial onde o processo de colonização teve grande influência na dispersão dos genes anormais, principalmente talassemias e falcemias. Assim, a distribuição das hemoglobinas anormais, provenientes de formas variantes e talassemias, está relacionada com as etnias que compõem nossa população. Dentre as hemoglobinas variantes, as mais freqüentes na população brasileira são a hemoglobina $S$ ( $\mathrm{HbS})$ e $\mathrm{C}(\mathrm{HbC})$, ambas de origem africana, mostrando a intensa participação do negro na composição populacional brasileira $(3,4,5)$. As talassemias são mais freqüentes em regiões que tiveram maior participação da colonização italiana $(4,6)$. Outras variantes raras como as hemoglobinas $D, J, I, N, G$, são encontradas em diferentes localidades (7).

As alterações que envolvem genes estruturais promovem a formação de moléculas de hemoglobinas com características bioquímicas diferentes das hemoglobinas normais, por isso são denominadas variantes, em sua maioria originada por substituições de aminoácidos resultantes de mudanças nas seqüências de nucleotídeos. As mutações afetando genes reguladores promovem desequilíbrio do conteúdo quantitativo das cadeias e, consequentemente, dos tipos normais de hemoglobinas, causando as talassemias $(8,10)$.

As talassemias do tipo alfa podem ter causas: hereditárias e adquiridas. Evidentemente as formas hereditárias são as mais comuns e atingem, pelo menos $4 \%$ da população brasileira. As formas adquiridas são secundárias a um processo patológico primário $(2,11,12)$. As talassemias do tipo beta constituem um grupo de alterações genéticas da síntese de hemoglobina extremamente diverso com variabilidade clínica em relação à sintomas e manifestações; condições resultantes de fatores genéticos e adquiridos $(2,13)$.

Considerando que todas essas hemoglobinopatias determinam importantes manifestações clínicas, cabe ressaltar a importância do diagnóstico laboratorial precoce destas patologias, evitando as conseqüências deletérias da doença.
Estudos populacionais permitem 0 diagnóstico de heterozigotos e 0 aconselhamento genético fornecendo subsídios para que os indivíduos decidam conscientemente sobre sua prole, além da melhoria na qualidade de vida dos doentes (14). Neste contexto é possível realizar estudos preventivos de hemoglobinopatias, principalmente nas regiões em que a incidência dessas alterações alcança freqüências marcantes para a saúde pública, enfocando da melhor maneira a população e o tipo de hemoglobinopatia que a acomete (15, 16). A elaboração de programas preventivos requer o suporte de órgãos oficiais de saúde, treinamento de pessoal capacitado para diagnóstico, aconselhamento genético e clínico dos pacientes e casais de risco $(17,18)$.

No presente estudo objetivamos avaliar as metodologias disponíveis para o diagnóstico laboratorial de hemoglobinopatias, comparando as análises de hemoglobina em diferentes populações e elaborar um esquema de programa preventivo de hemoglobinopatias segundo a resposta de cada população analisada.

\section{Casuística e métodos}

As populações analisadas foram: estudantes, portadores de anemia a esclarecer, doadores de sangue e recém-nascidos. 0 grupo dos estudantes foi escolhido como grupo controle. Todas as amostras de sangue foram colhidas com anticoagulante e após prévia autorização dos participantes, conforme orientação do Comitê de Ética em Pesquisa.

A metodologia empregada para as análises foram: Testes Seletivos - resistência globular osmótica em solução de cloreto de sódio a 0,36\% (19), eletroforese em $\mathrm{pH}$ alcalino em acetato de celulose (20), análise da morfologia eritrocitária à fresco (2).

Para a confirmação dos diagnósticos foram realizados testes específicos como: eletroforese quantitativa em acetato de celulose, $\mathrm{pH}$ 8,5 para dosagem de hemoglobina $A$ (20), eletroforese de diferenciação em ágar-fớsfato pH 6,2 (21) para diferenciação das variantes S e C; dosagem de hemoglobina fetal (22), pesquisa de corpos de Heinz e agregados intra-eritrocitários de hemoglobina $\mathrm{H}$ (23). 


\section{Resultados}

No período de setembro de 1999 à janeiro de 2000 foram analisadas amostras de sangue de diferentes grupos populacionais e os resultados obtidos nas análises de hemoglobinas podem ser observados na Tabela 1. Estatisticamente os valores de hemoglobinas normais e anormais entre os diferentes grupos populacionais estudados, apresentaram diferença entre estudantes e recém-nascidos com valores de $p=1,0000$, considerado não significativo. A diferença entre os valores encontrados para os estudantes, tido como grupo controle, e portadores de anemia a esclarecer, mostrou valor de $p=0,0157$, sendo considerado significativo. Entre estudantes e doadores de sangue a diferença encontrada mostrou valor de $p<0,0001$, sendo considerado extremamente significativo.

No grupo dos recém-nascidos foram analisados um total de 100 amostras de sangue de cordão umbilical. A origem racial destes indivíduos não pode ser avaliada pois não foi fornecida adequadamente pelo hospital. 0 valor médio de hemoglobina fetal encontrado foi de $81,71 \%$, com um valor mínimo de $62,85 \%$ e um valor máximo de $96 \%$. As taxas de normalidade da hemoglobina fetal para indivíduos nesta faixa etária varia de 70 à $90 \%$. Com relação às hemoglobinopatias, 24 (24\%) recém-nascidos apresentaram hemoglobinas alteradas, sendo 10 (10\%) indivíduos com valores de hemoglobina fetal superiores à $90 \%$ sugerindo beta talassemia heterozigota, $10(10 \%)$ indivíduos com valores de hemoglobina fetal dentro dos padrões de normalidade e presença de hemoglobina $\mathrm{H}$ / Bart's, sugerindo alfa talassemia heterozigota. Um (1\%) indivíduo apresentou hemoglobina $\mathrm{H}$ / Bart's e valores de hemoglobina fetal superiores à $90 \%$, sugerindo interação de alfa talassemia com beta talassemia heterozigota. Três (3\%) indivíduos apresentaram hemoglobina $\mathrm{S}$ que, pela sua concentração, sugere traço falcêmico. A Tabela 2 apresenta cada um dos tipos de hemoglobinas anormais encontrados em cada grupo estudado.

No grupo dos portadores de anemia à esclarecer, foram analisadas amostras de sangue de cinqüenta $(100 \%)$ pacientes com idade variando de um a 58 anos. Três (6\%) indivíduos eram caucasóides, 5 (10\%) não-caucasóides. Em $42(84 \%)$ indivíduos a origem racial não foi informada pelos laboratórios que encaminharam as amostras. 0 grupo de não-caucasóides incluiu negros, mulatos, e amarelos. Com relação à hemoglobinopatias, observamos 13 (26\%) indivíduos com alfa talassemia, $7(16 \%)$ com beta talassemia heterozigota, $1(2 \%) \mathrm{com}$ interação alfa/beta talassemia e $1(2 \%)$ com hemoglobina $A B 2$; uma variante de cadeia delta sem alterações fisiológicas; totalizando 22 (40\%) indivíduos com hemoglobinas alteradas. Não foi encontrado nenhum portador de traço falcêmico, devido ao fato desta herança em heterozigose não provocar anemia.

Tabela 1. Valores de hemoglobinas normais e anormais encontrados em cada grupo populacional estudado

\begin{tabular}{l|c|c|c}
\hline Grupo estudado & $\begin{array}{c}\text { Hemoglobinas } \\
\text { normais N(\%) }\end{array}$ & $\begin{array}{c}\text { Hemoglobinas } \\
\text { anormais N(\%) }\end{array}$ & $\begin{array}{c}\text { Total } \\
\text { N(\%) }\end{array}$ \\
\hline Recém - nascidos & 76 & 24 & 100 \\
& $76 \%$ & $24 \%$ & $100 \%$ \\
\hline Portadores de anemia a esclarecer & 28 & 22 & 50 \\
& $56 \%$ & $44 \%$ & $100 \%$ \\
\hline Doadores de sangue & 249 & 13 & 262 \\
& $95,03 \%$ & $4,96 \%$ & $100 \%$ \\
\hline Estudantes & 84 & 28 & 112 \\
& $75 \%$ & $25 \%$ & $100 \%$ \\
\hline Total & 437 & 87 & 524 \\
\hline
\end{tabular}


Entre o grupo de doadores de sangue, foram analisadas 262 amostras de indivíduos com média de idade de 34,21 anos, onde a idade mínima foi de 18 anos e a máxima 64 . Com referência a origem racial, 203 (74,48\%) eram caucasóides e 59 (22,52\%) não-caucasóides. Para a análise de hemoglobinas anormais, 249 (95,03\%) indivíduos apresentaram padrão eletroforético normal para hemoglobinas e 13 (4,96\%) apresentaram hemoglobinas anormais, dentre os quais, dois $(0,76 \%)$ com hemoglobina AS, $3(1,14 \%)$ com hemoglobina AC, 5 (1,90\%) com suspeita de alfa talassemia, $1(0,38 \%)$ com suspeita de beta talassemia e $2(0,76 \%)$ com hemoglobina $A B 2$. Para este grupo em especial, correlacionamos a presença de hemoglobinas anormais segundo a origem racial que está demonstrada na Tabela 3. Dentre os caucasóides, $2(0,76 \%)$ apresentaram hemoglobina AS, 2 (1,14\%) hemoglobina AC, 5 $(1,90 \%)$ alfa talassemia, $1(0,38 \%)$ beta talassemia. Entre os não-caucasóides encontramos 1 (0,38\%) indivíduo com hemoglobina AC e $2(0,76 \%)$ com hemoglobina $A B 2$.

A análise estatística para presença ou não de hemoglobinas anormais e origem racial em doadores de sangue mostrou valor de $p=1,0000$, considerado não significativo.

No grupo dos estudantes foram realizadas análises para hemoglobinopatias em 112 amostras de sangue de indivíduos cuja média

Tabela 2. Tipos de hemoglobinas encontradas nas populações analisadas

\begin{tabular}{l|c|c|c|c|c|c|c}
\hline \multirow{2}{*}{ População } & \multicolumn{7}{c}{ Tipos de hemogl obinas } \\
\cline { 2 - 8 } & Hb AA & Hb S & Hb C & Alfa-tal & Betatal & Outros & Total \\
\hline R.N. & 76 & 3 & & 10 & 10 & 1 & 100 \\
$(100)$ & $(76 \%)$ & $(3 \%)$ & - & $(10 \%)$ & $(10 \%)$ & $(1 \%)$ & \\
\hline $\begin{array}{l}\text { Portadores de anemia } \\
\text { (50) }\end{array}$ & 28 & & & 13 & 7 & 2 & 50 \\
$\begin{array}{l}\text { Doadores de sangue } \\
\text { (262) }\end{array}$ & $\begin{array}{c}249 \\
(56 \%)\end{array}$ & - & - & $(26 \%)$ & $(16 \%)$ & $(4 \%)$ & \\
\hline $\begin{array}{l}\text { Estudantes } \\
(112)\end{array}$ & 2 & 3 & 5 & 1 & 2 & 262 \\
\hline
\end{tabular}

Tabela 3. Tipos de hemoglobinas segundo a origem racial em doadores de sangue

\begin{tabular}{l|c|c|c|c|c|c|c}
\hline \multirow{2}{*}{ Indivíduos } & \multicolumn{7}{c}{ Tipos de hemoglobinas } \\
\cline { 2 - 8 } & Hb AA & Hb AS & Hb AC & Alfa - tal & $\begin{array}{c}\text { Beta-tal } \\
\text { het }\end{array}$ & Outros & Total \\
\hline Caucasóides & 193 & 2 & 2 & 5 & 1 & 0 & 203 \\
& $73,66 \%$ & $0,76 \%$ & $1,14 \%$ & $1,90 \%$ & $0,38 \%$ & $0 \%$ & $77,48 \%$ \\
Não Caucasóides & -56 & 0 & 1 & 0 & 0 & 2 & 59 \\
& $21,37 \%$ & $0 \%$ & $0,38 \%$ & $0 \%$ & $0 \%$ & $0,76 \%$ & $22,51 \%$ \\
\hline Total & 249 & 2 & 3 & 5 & 1 & 2 & 262 \\
\hline
\end{tabular}

$\mathrm{Hb} A \mathrm{~A}$ : hemoglobinasnormais

Hb AS: traço falcêmico (indivíduo heterozigoto)

$\mathrm{Hb}$ AC: hemoglobina C (in divíduo heterozigoto)

Beta - tal het: beta talassemia heterozigota

Alfa - tal: alfa - talassemia

Outros: hemoglobina $A B_{2}$ 
Tabela 4. Tipos de hemoglobinas segundo a origem racial no grupo de estudantes

\begin{tabular}{l|c|c|c|c|c|c}
\hline \multirow{2}{*}{ Indivíduos } & \multicolumn{7}{|c}{ Tipos de hemoglobinas } \\
\cline { 2 - 7 } & Hb AA & Hb S & Alfa - tal. & $\begin{array}{c}\text { Beta-tal. } \\
\text { het. }\end{array}$ & $\begin{array}{c}\text { Alfa/ beta } \\
\text { tal. }\end{array}$ & $\begin{array}{c}\text { Total } \\
\mathbf{1 1 2} \mathbf{- 1 0 0 \%}\end{array}$ \\
\hline Caucasóides & 80 & 1 & 12 & 9 & 2 & 104 \\
& $71,42 \%$ & $0,89 \%$ & $10,71 \%$ & $8,03 \%$ & $1,78 \%$ & $92,8 \%$ \\
Não - caucasóides & 5 & 0 & 2 & 1 & 0 & 8 \\
& $4,64 \%$ & $0 \%$ & $1,78 \%$ & $0,89 \%$ & $0 \%$ & $7,14 \%$ \\
\hline
\end{tabular}

Hb AA: hemoglobinas normais

$\mathrm{Hb}$ S: hemoglobina S

Alfa - tal: alfa - talassemia

Beta - tal het: beta talassemia heterozigota

Alfa / beta tal: interação alfa / beta talassemia

Tabela 5. Hemoglobinas anormais em doadores de sangue e estudantes, segundo a origem racial

\begin{tabular}{l|c|c|c}
\hline & Estudantes & Doadores de sangue & Total \\
\hline Caucasóides & 24 & 10 & 34 \\
& $60 \%$ & $25 \%$ & $85 \%$ \\
Negróides & 3 & 3 & 6 \\
& $8 \%$ & $8 \%$ & $15 \%$ \\
Total & 27 & 13 & 40 \\
& $68 \%$ & $33 \%$ & $100 \%$ \\
\hline
\end{tabular}

$\mathrm{p}=0.3699$

Odds Ratio" $=2.400$

Intervalo de confiança de 95\%: $0.4118<$ <á < 13.986 (usando a aproximação de Woolf).

de idade foi de 22,39 anos, sendo 18 anos a idade mínima e 45 a máxima. Segundo a origem racial, 104 (92,85\%) eram caucasóides e 8 $(7,14 \%)$ eram não-caucasóides.

Neste grupo, encontramos 1 (0,89\%) indivíduo com hemoglobina AS sugerindo traço falcêmico, $14(12,5 \%)$ com alfa talassemia, 10 $(8,93 \%)$ com beta talassemia e $2(1,78 \%)$ com interação alfa/beta talassemia, totalizando 27 (25\%) indivíduos com hemoglobinas alteradas.

A Tabela 4 apresenta os tipos de hemoglobinas encontradas segundo a origem racial para o grupo de estudantes, evidenciando que do total de 104 (92,8\%) indivíduos caucasóides, $80(71,42 \%)$ apresentaram hemoglobinas normais, $1(0,89 \%)$ com hemoglobina $\mathrm{S}$ sugerindo traço falcêmico, 12 $(10,71 \%)$ indivíduos com alfa talassemia, 9
$(8,03 \%)$ com beta talassemia e $2(1,78 \%)$ com interação de alfa com beta talassemia. Entre os não-caucasóides que totalizaram 8 (7,14\%) indivíduos, encontramos $5(4,64 \%)$ indivíduos com hemoglobinas normais, 2 (1,78\%) com alfa talassemia, $1(0,89 \%)$ com beta talassemia. A análise estatística mostrou valor de $p=0,3969$, considerado não significativo.

Quando comparamos a presença de hemoglobinas anormais entre doadores e estudantes, segundo a origem racial, encontramos valor de $p=0,3699$, considerado não significativo (Tabela 5 ).

No grupo dos estudantes, através dos testes utilizados na triagem de hemoglobinopatias, observamos a presença de prováveis quadros anêmicos. Desta forma, além dos 27 prováveis portadores de alteração de hemoglobinas, 11 
apresentaram suspeita de anemia carencial. Os 38 indivíduos foram chamados para avaliação clínica onde foram solicitados exames complementares como hemograma, ferro sérico, ferritina e transferrina para afastar ferropenia.

Através da análise de hemoglobinas em diferentes grupos populacionais, observamos que a metodologia laboratorial proposta oferece subsídios para a correta avaliação das diferentes formas de hemoglobinas anormais, cujo diagnóstico laboratorial preciso dependerá da combinação dos métodos aplicados na triagem e confirmação dos suspeitos. As Figuras 1 e 2 ilustram os procedimentos eletroforéticos em $\mathrm{pH}$ alcalino e ácido, onde se fundamenta o diagnóstico das principais hemoglobinopatias.

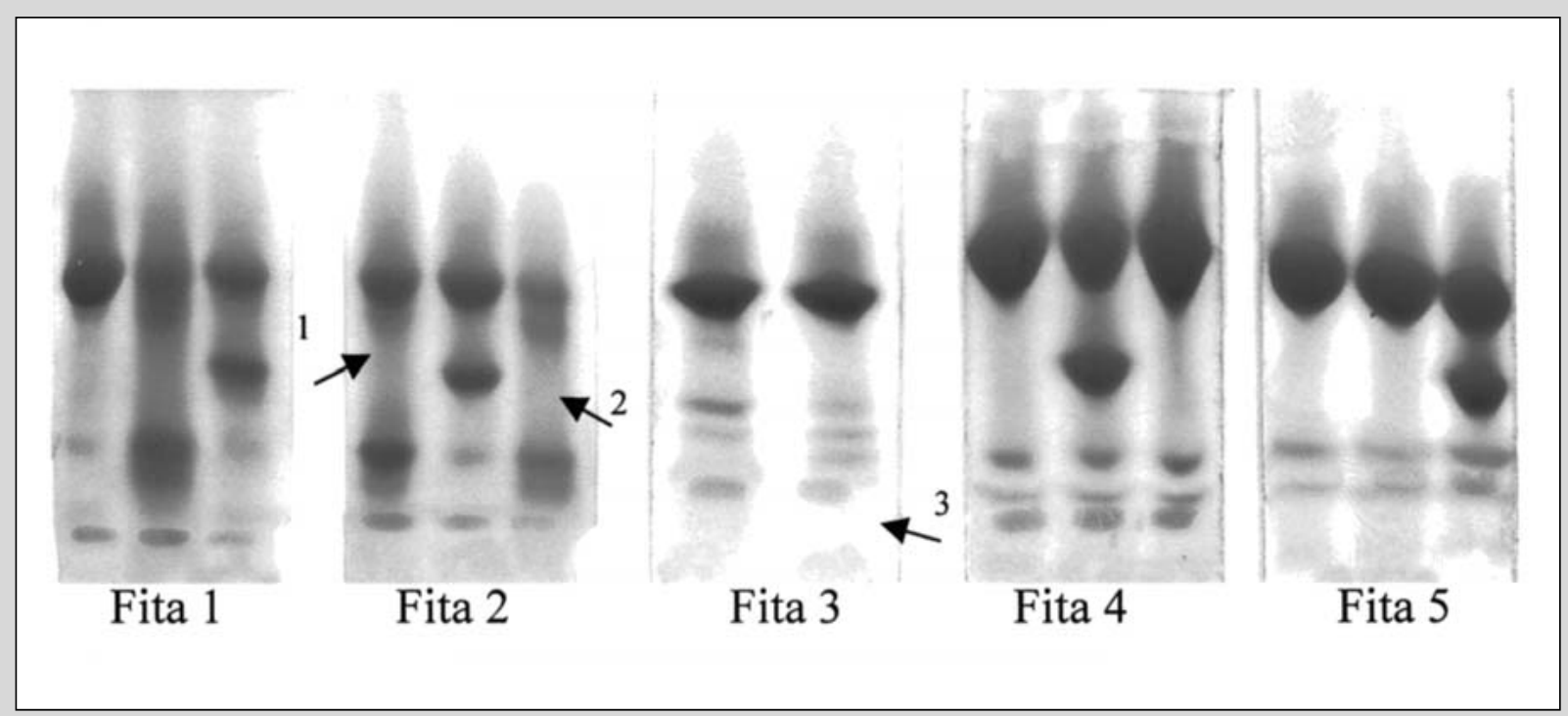

Figura 1. Eletroforese em acetato de celulose $\mathrm{pH} 8,6$

Observação: as setas na fita 2 indicam a presença de metahemoglobina, devido ao en velhecimento de algumas amostras. Na fita 3, a seta indica a presença da mutante de cadeia delta $A B_{2}$
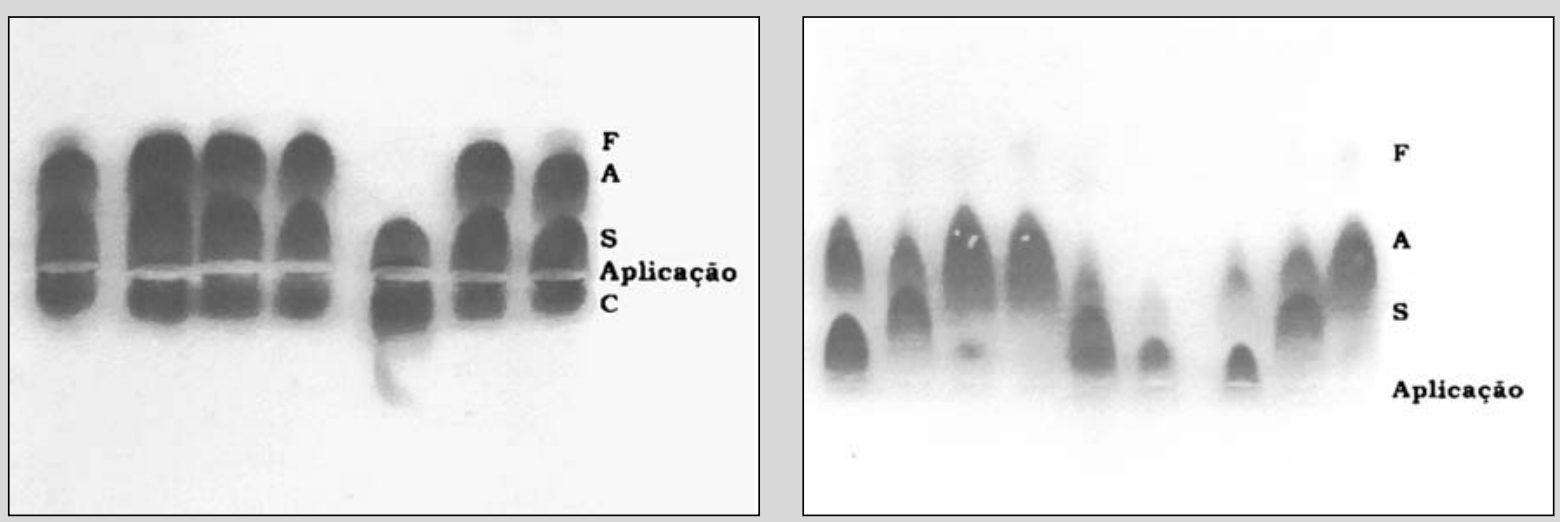

Figura 2. Eletroforese em pH 6,2 ágar - fosfato para diferenciação

F: Hemoglobina Fetal

A: Hemoglobina A

S: Hemoglobina S 


\section{Discussão}

A população brasileira caracteriza-se por apresentar grande heterogeneidade genética, derivada da contribuição dos seus grupos raciais formadores, por si também já muito diversificados, e dos diferentes graus com que eles se intercruzaram nas várias regiões do país (24). A miscigenação da população brasileira é resultante das imigrações que ocorreram no período da colonização compreendido entre os séculos XVI e XIX, nos quais o Brasil recebeu milhões de imigrantes europeus e asiáticos. A freqüência das anemias hereditárias reflete a diversidade de origens raciais e os diferentes graus de mistura entre brasileiros de cada região do país. As hemoglobinopatias, especialmente síndromes falcêmicas, hemoglobina $\mathrm{C}(\mathrm{Hb} \mathrm{C})$ e talassemias são comuns no Brasil com prevalências diferenciadas $(25,26,27,28,29,30)$.

A Organização Mundial de Saúde recomenda a implantação de programas para prevenção e controle de hemoglobinopatias na América Latina, especialmente no Brasil $(31,32)$. A organização de um programa preventivo requer suporte dos órgãos oficiais de saúde, treinamento de pessoal capacitado para diagnóstico, aconselhamento genético e clínico dos pacientes (18). 0 sucesso destes programas depende da receptividade, disponibilidade e interesse da população a ser estudada (33).

A detecção de indivíduos portadores das formas imperceptíveis de hemoglobinopatias, os heterozigotos, são extremamente importantes para a saúde pública pois, além de representarem fonte de novos heterozigotos, podem, através de casamentos entre portadores, originar indivíduos homozigotos e duplos heterozigotos como, por exemplo, os portadores de hemoglobina $\mathrm{SC}$ ( $\mathrm{Hb} \mathrm{SC}$ ), que manifestam uma forma clínica. Os indivíduos homozigotos diagnosticados deverão ser devidamente encaminhados à orientação médica para tratamento precoce, minimizando as manifestações clínicas $(34,35)$. Dessa forma, destacamos que os pontos fundamentais de um programa preventivo de hemoglobinopatias compreende a divulgação da informação à população, o reconhecimento de heterozigotos, diagnóstico neonatal, e aconselhamento genético. Este compreende atividades de esclarecimento ao portador da alteração de maneira clara devendo ser realizado por especialista ou geneticista com material informativo específico (2).

Os programas de prevenção colaboram para a conscientização dos heterozigotos fornecendo informações para que possam decidir responsavelmente sobre o futuro de seus descendentes; favorece o direcionamento clínico nos casos mais brandos e indica 0 acompanhamento adequado aos portadores das formas graves da doença. É importante uma tecnologia adequada para o diagnóstico, utilizando vários testes laboratoriais seletivos e de confirmação, dados clínicos e estudo familial, bem como a formação de pessoal capacitado para o diagnóstico laboratorial correto.

No presente estudo, constatamos que no grupo dos recém-nascidos, onde foram analisadas 100 amostras de sangue de cordão, verificamos que 24 (24\%) amostras sugeriram portadores de hemoglobinas anormais. Comparados ao grupo dos estudantes, com $27(24,11 \%)$ portadores de hemoglobinas anormais, a análise estatística não apresentou diferença significativa e, os valores encontrados refletiram o esperado pois são populações gerais. Em recém-nascidos, a hemoglobina fetal será substituída pela hemoglobina A por volta dos seis meses de idade, período no qual novos testes deverão ser realizados para confirmar as suspeitas de hemoglobinas anormais, em especial nas mutantes de cadeia beta.

Entre estudantes e doadores de sangue, os valores de hemoglobinas anormais encontrados revelaram uma diferença extremamente significativa, a qual foi de que 27 (7\%) estudantes portadores de hemoglobinas anormais e 13 (3\%) doadores com hemoglobinas anormais. Esse resultado deve-se à triagem previamente realizada para doadores de sangue, o que os pré-seleciona principalmente para as formas de hemoglobinopatias que afetam os valores hematimétricos, e os estudantes são chamados ao acaso. A freqüência de estudantes suspeitos de apresentarem anemia carencial em nosso 
grupo, pode ser explicada devido à condição física e psicológica em que se encontravam no período da coleta, o qual coincidiu com época de provas finais, onde não mantinham alimentação adequada e estavam em relativo estresse.

No grupo dos portadores de anemia, encontramos 22 (44\%) indivíduos com hemoglobinas anormais, num total de 50 amostras de sangue analisadas. Tal fato pode ser explicado pelos portadores de anemia constituírem um grupo com manifestações clínicas de anemia à esclarecer.

0 modelo de programa preventivo que usamos contou com apoio de equipe de psicólogos que atuou na abordagem dos estudantes interessados, fornecendo informações sobre as hemoglobinopatias. Dessa forma participaram conscientemente do estudo mediante autorização prévia, segundo orientação do Comitê de Ética em Pesquisa com Seres Humanos.

Os resultados foram encaminhados aos estudantes e naqueles onde observamos a presença de alterações de hemoglobinas, fornecemos material específico e orientação adequada para avaliação clínica, testes complementares e estudo familiar. Nesta fase, a atuação da psicóloga da equipe foi fundamental, minimizando ansiedades e algumas dúvidas que pudessem surgir no momento de uma nova coleta e estudo da família, evidenciando a importância da atuação multiprofissional em programas preventivos de alterações genéticas para que obtenhamos 0 sucesso esperado.

\section{Conclusões}

O presente estudo procurou mostrar a importância do diagnóstico laboratorial de hemoglobinopatias, bem como as atividades de prevenção que permitem 0 aconselhamento genético, o tratamento precoce nos casos de homozigose e sugerir a realização de uma triagem mais específica em doadores de sangue.

Em análises realizadas nas diferentes populações propostas observamos a importância do estudo populacional, que permite uma visualização da distribuição das hemoglobinopatias na população humana, auxiliando na elaboração de programas preventivos.

Podemos auxiliar a comunidade promovendo campanhas de esclarecimento nas escolas e em serviços de saúde da rede pública e particular, nas quais se faz necessário o diagnóstico de indivíduos heterozigotos, 0 aconselhamento genético e o diagnóstico neonatal.

Nas avaliações em neonatos, os resultados encontrados sugerem a implantação de serviços de esclarecimento à população que realmente atinjam a comunidade como um todo, além de fornecer o tratamento de suporte aos indivíduos afetados.

Pela análise dos portadores de anemia à esclarecer, concluímos que existe uma grande necessidade de aumentar a capacidade de formação de recursos humanos para o correto diagnóstico laboratorial.

Um programa preventivo necessita da colaboração de diversos profissionais para que as informações necessárias sejam reunidas. Notamos que, devido ao padrão de herança das anemias, é necessário informar a origem racial dos indivíduos e nem todos os serviços prestam tal informação, o que dificulta uma análise mais detalhada de cada caso.

No grupo dos doadores de sangue 0 resultado mostra que das 262 amostras estudadas, 13 apresentaram algum tipo de alteração de hemoglobina. A preocupação em torno dos doadores de sangue se deve ao fato de que, o sangue doado é transfundido para indivíduos debilitados que necessitam de sangue com capacidade de transportar oxigênio para as áreas carentes do organismo. Os doadores heterozigotos são indivíduos que não apresentam sintomatologia clínica evidente, e por isso se candidatam à doação. Devemos considerar o risco de uma transfusão de sangue de um indivíduo portador de hemoglobina S para um receptor também heterozigoto para siclemia. Os efeitos indesejáveis se devem ao potencial de falcização do receptor bem como às alterações do produto hemoterápico em conseqüência do processamento e estocagem do sangue (36). 
Entre os estudantes, das 112 amostras analisadas, encontramos 28 indivíduos que apresentavam algum tipo de alteração. A abordagem destes estudantes para contribuírem com o presente estudo foi realizado com auxílio psicológico, que procurou esclarecer o que é anemia hereditária, explicando suas principais conseqüências e a importância de um diagnóstico preventivo, além de atuar minimizando as ansiedades dos alunos. Uma vez sendo estudantes universitários e esclarecendo o tipo de alterações que apresentam, podemos concluir que a prevenção de hemoglobinopatias é importante para detecção dos heterozigotos e esclarecêlos a respeito da alteração que possuem. Sendo assim, estaremos contribuindo para com 0 conhecimento sobre esta alteração genética e reduzindo morbidade e mortalidade dos indivíduos com a forma grave da doença.

\section{Laboratory diagnosis of Hemoglobinopathies in different population groups}

Giselda M. Orlando, Paulo C. Naoum, Fatima A. M. Siqueira, Cláudia R. Bonini-Domingos

\section{Abstract}

The inherited haemoglobinopathies are a heterogen eous group of recessive disorders that include the thalassaemias and sickle cell disease. Nearly a thou sand mutant alleles have now been characterized. The mutations are regionally specific and in most cases the geographical and ethnic distributions have been determined providing the foundation for a program of control through screening, genetic counseling and prenatal diagnosis. The diagn osis of hemogl obin nopathies requires care for the methodologies applied and the population group which will be evaluated. The information about the abnormal hemoglobin, the medical and psychological aspects and genetic counseling of the carriers and their families are goals of great importance for the success of preventive programs in this area. Aiming to evaluate the laboratory methods for hemoglobinopathy screening and their use in clinical laboratories, we have compared abnormal hemoglobins incidence in the different population groups: blood donors, anemia carriers, newborn and students. The laboratory methods applied involved eletrophoretic proceedings, cytological and biochemical analysis. Within the period from September 1999 through January 2000, we analyzed 524 individuals with varied types of abnormal hemoglobins. Among blood donors, we diagnosed two sickle cell carriers, which suggest the necessity for better care in the process of selection of blood donor candidates. The current interest in the medical and social aspects of sickle cell anemia has resulted in a great increase in methodology research leading to the development of sickle cell screening techniques.

Rev.bras. hematol. hemoter., 2000, 22(2) : 111-121

Key-words: hemoglobin opathies prevention, laboratory diagnosis, abnormal hemoglobin, thalassemias

\section{Referências Bibliográficas}

1. Thompson, M.W., Mclnnes, R.R., Willard, H.F. Genética médica 5.ed. Rio de Janeiro: Guanabara Koogan, 1993.

2. Bonini-Domingos, C.R.. Prevenção das hemoglobinopatias no Brasil: diversidade gen ética e metodologia laboratorial. São José do Rio Preto, 1993. Tese (Doutorado em Ciências Biológicas) - Instituto de Biociências, Letras e Ciências Exatas, Universidade Estadual Paulista.

3. Naoum, P.C., Machado, P.E.A., Michelin, O.C. , Cury, R.R., Pio da Silva, M. Concentration of haemoglobin $A$ and Fetal in Brazilian Indians relationshîp between these haemoglobins and malaria. Cienc. Cult, v.31, p.188-190, 1979.

4. Naoum, P.C. Anemiasimigrantes: a origem dasanemiashereditáriasno Brasil. Cienc. Hoje, v.3, n.14, p.59-64, 1984.

5. Álvares Filho, F., Naoum, P.C., Moreira, H.W., Ângulo, I.L. Variabilidade polimórfica das hemoglobinas humanas anormais em indivíduos das cidades de Barretos e Colina, SP, Brasil. Rev. Bras. Patol. Clin., v.24, n.2, p.32, 1988. 
6. Ramalho, A.S. A talassemia minor como causa de anemia no estado de São Paulo. Rev. Bras. Patol. Clin., v.22, p.32-38, 1986 b.

7. Naoum, P.C., Álvares Filho, F., Domingos, C.R. B.6, Ferrari, F., Moreira, H.W., Sampaio, Z.A., Maziero, P.A., Castilho, E. M. Hemoglobinas anormais no Brasil: prevalência e distribuição geográfica. Rev. Bras. Patol. Clin., v.23, n.3, p.6872, 1987a.

8. Naoum, P.C. Diagnóstico de hemoglobinopatias. São Paulo: Sarvier., 1987 b.

9. Edelstein, S.J. The sickled cell: from myths to molecules. Cambridge: Harvard University Press, 1996.

10. Weatherall, D.J., Wainscoat, J.S. The molecular pathology of thalassemia. In: Hoffbrand, A.V. (Ed.) Recent advances in haematology. Oxford: Churchill Livingstone, 1985 . p.63-88.

11. Naoum, P. C. Eletroforese técnicas e diagnósticos. São Paulo: Santos, 1999.

12. Weatherall, D.J., Clegg, J.B. The talassemias syndromes. 3.ed. Oxford: Blackwell Scientific publication, 1981.

13. Beutler, E. The common anemias: rewiew. J. Amer. Med. Assoc., v. 259, n. 16, p. 2433 - 2437, 1988.

14. Bianco, I., Graziani, B., Lerone M., Congedo, P., Aliquo, M.Q., Ponzini, D., Branconi, F., Foglietta, E., Mondiano, G., Silvestroni, E. La prevenzione dell' anemia mediterrânea nel Lazio: risultati del programa aplicatto negli ultimi 10 anni. Prog. Med. Roma, v.42, p. 521-549, 1986.

15. W.H.O. World Health Organization. In: Annual Meeting of the WHO, 6, 1989. Cagliari. Report: working group on the feasibility study on hereditary disease community control programes (hereditary anaemies).

16. Ramalho, A.S. As hemoglobinopatias hereditárias: um problema de saúde pública no Brasil. São Paulo: FCA, 1986 a.

17. Fessas, P. Prevention of thalassemia and haemoglobin S Syndromes in Greece. Hemogl. Proc. Int. Meeting London, v.78, p.168-172, 1987.
18. Cao, A., Rosatelli, C., Pirastu, M., Galanello, R. Thalassemias in Sardinia: molecular pathology phen othype-gen otype correlation and prevention. Am. J. Pediatr. Hematol. Oncol., v.13, n.2, p.179-188, 1991.

19. Silvestroni, E., Bianco, I. Screening for microcytemia in Italy: analysis of data collected in the past 30 years. Am. J. Hum. Genet, v.27, p.198, 1975.

20. Marengo-Rowe, A.J. Rapid electrophoresis and quantitation of haemoglobin on cellulose acetate. J. Clin. Pathol., v.18, p.790-92, 1965.

21. Vella, F. Acid agar gel electrophoresis of human hemoglobins. Am. J. Clin. Pathol., v.49, p.440, 1968.

22. Betke, K., Marti, N.R., Schlicht, I. Estimation of small percentages of foetal haemoglobin. Nature, v.184, p.1877, 1959.

23.Papayannopoulos, R., Stamatayannopoulos, G. Stains for inclusions bodies. In: Standartization of laboratory reagents and methods for detection of haemoglobinopathies. Atlanta: Hew Publications, 1974.

24. Naoum, P.C. Hemoglobinopatias no estado de São Paulo: métodos de estudo, prevalência, distribuição geográfica, relações históricas e antropológicas. São José do Rio Preto, 1982. Tese (Livre Docência) - Instituto de Biociências, Letras e Ciências Exatas, Universidade Estadual Paulista.

25. Salzano, F.M., Tondo, C.V. Hemoglobin types in brazilian population. Hemoglobin, v. 6, p. 85 - 97, 1982.

26. Naoum, P.C., Cury, P.R. Hemoglobinopatias no estado de São Paulo: distribuição geográfica e polimorfismos. Bol. Sanit. Panam., v. 101, p. 88 - 95, 1983 a.

27. Naoum, P.C. et al. Hemoglobinas anormais em uma população do estado de São Paulo, Brasil. Rev. Bras. Patol. Clin., v. 19, n. 3, p.86 - 89, 1983 b.

28. Zago, M.A., Costa, F.F. Hereditary haemoglobin desorders in Brazil. Trans. R. Soc. Med. Hyg., v. 79, p. 385 - 388, 1985.

29. Teixeira, R.C., Ramalho, A.S. Genetics and public health: response of a Brazilian population to an optional hemoglobinopathy program. Rev. Bras. 
Genet., v. 17, n. 4, p. 435 - 438, 1994.

30. Sonati, E. etal. Hereditary hemoglobinopathies in a population from Southeast Brazil. Hemoglobin, v. 20, n. 2, p. 175 - 179, 1996.

31. W.H.O. Memorandum from a W. H. O. meeting Bull: W.H.O., v. 61, p. 63 - 80, 1983.

32. Penchszadeh, V. Genetics services for hemoglobinopathies in Latin- America. Joint W.H.O. In: Meeting on Prevention and Control of Hemoglobinopathies. Nicosia, Cyprius, 1993.

33. Bowman, J.E. Prenatal screening for hemoglobin opathies. Am. J. Hum. Genet, v. 48, n.3, p. 433-438, 1991.

34. Barnhart, M.I., Henry, R.L., Lusher, S.M.

Sickle cell. U.S.A: Scope, 1976.
35. Silvestroni, E., Bianco, I., Graziani, B., Carbonl, C., D'arca, S.U. First screening of thalassaemia carriers in intermediate schools in Latium. J. Med. Genet., v. 15, p. 202 - 207, 1978.

36. Nascimento, M.L.P., Borja, M.M.P. Portadores de hemoglobina S: um estudo comparativo entre vários serviços de hemoterapia brasileiros e o impacto médico- social em Salvador, Bahia. Rev. Bras. Hematol. Hemoter., v.21, n.2, p.6771, 1999.

Recebido: 09/02/00

Modificado: $10 / 04 / 00$

Aceito: $10 / 06 / 00$ 